\title{
Lagrangian drifter paths and length scales in the tropical Pacific warm pool from 1990 to 1991: with application of fractal techniques
}

\author{
S. Nakamoto ${ }^{1,2}$, Y. Kashino, ${ }^{1}$, . Fang ${ }^{1,3}$, T. Kawano', K. Muneyama ${ }^{1}$ and T. Nakanishi ${ }^{1}$ \\ 'Japan Marine Science and Technology Center, 2-15, Natsushima-cho, Yokosuka, 237, Japan \\ ${ }^{2}$ Sanyo Hydrographic Survey Co, Ltd., 3-17, Nihonbashi-Horidomecho-1-chome, Chuouku, Tokyo, 103, Japan \\ ${ }^{3}$ First Institute of Oceanography, State Oceanic Administration Quingdao, People's Republic of China
}

Received 1 December 1993 - Accepted 2 March 1994 - Communicated by A.D. Kirwan

\begin{abstract}
This paper presents an analysis of WOCE/TOGA surface drifter paths and its interpretation in conjunction with the west Pacific warm pool water motion. Our interest here lies in the existence of scale invariance in the observed data sets. The analysis proceeds by detecting scale invariance in the drifter paths data, and interpreting the invariance in terms of the statistical second order moment. The range of constant scaling exponent was found to be between 5 days and 10 days, and this range corresponded with the "long tail" of the temporal correlation function in the zonal direction. Velocity covariances in both the zonal and meridional directions were computed, and corresponding diffusivities were $8100 \mathrm{~m}^{2} / \mathrm{sec}$ meridionally and $41000 \mathrm{~m}^{2} / \mathrm{sec}$ zonally.

Considering the existence of large scale mean flow, it is thought that self-similar energy cascade processes associated with constant scaling exponent may be responsible for the anomalous zonal diffusivity, while the meridional diffusivity may be approximated by ordinary Brownian processes. We suggest that the scale invariance of the WOCE/TOGA surface drifter paths may be a manifestation of energy cascade processes from large scale mean flow to smaller scale irregular flow that is represented by fractional Brownian motion in the zonal direction.
\end{abstract}

\section{Introduction}

The oceanic surface drifter paths have been often used to describe a single realization of a water parcel path, which provide us with an informative Lagrangian trail of the flow excuting the nonlinear dynamics in the governing equations. However such data was not enough to obtain the overall flow structure in the space-time domain for the regime of the NavierStokes quations.

The notion of chaotic behavior of Lagrangian trajectories arises naturally because the Lagrangian equa- tions of motion define a low-order dynamical system. The motion of individual fluid parcels would be unpredictable beyond some short period of time. "Pessimists" have argued that the ocean system is so complex and nonlinear that the chaotic nature may prohibit predicting such long term varability in the ocean.

Osborne et al.[1989] studied the fractal and scaling properties of the Lagrangian trajectories of the three satellite tracked, freely drifting buoys placed in the Kuroshio extension region. They found that for space scales extending from at least 20 to $150 \mathrm{~km}$ and time scale from 1.5 days to 1 week, each of the trajectories displays fractal and scaling behavior.

Sanderson and Booth [1991] analyzed drifter data from the Northeast Atlantic and found that single particle motion has fractal dimension $\mathrm{D}=1.28$ at scales 5 to $100 \mathrm{~km}$. Using fractional Brownian motion they modeled the single particle trajectories to show the time dependent variance (particle dispersion) as $t^{3 / 2}$. They also showed that the two-particle motions was modeled as an accelerated fractional Brownian motion in which the relative speed between particles increases with the time elapsed since the two particles were infinitesimally near each other. Using this approach they examined how measurements of eddy-dispersion and integral time scale/length scale depend upon how the drifter tracking measurements are made.

The recent development of low-cost Lagrangian drifters that faithfully follow water particle motion to within 1 $\mathrm{cm} / \mathrm{sec}$ [Niiler et al. 1987] offered us the opportunity to measure the response of the upper ocean on a horizontal scale larger than historically observed eddies in the area and comparable to the wind response of the ocean [McNally 1981]. The drifter data set covers a significant area of the ocean, averaging over many mesoscale features, so it may be an ideal observational tool for large scale oceanic motion. The implication of our analysis is that large scale mean surface flow in the west Pacific warm pool region advects heat in the mixed layer. Therefore, the role of ocean surface circulation in maintaining the distribution of surface temperatures will have to be revealed by analyzing Lagrangian path data. 
In this study we focus our attention on quantifying scale invariance of the surface flow field in the west $\mathrm{Pa}$ cific warm pool region by using WOCE/TOGA Surface Velocity Program (SVP) drifter path data. We are interested in the description of the flow in and out of the pool to arrive at the length representing the persistent isotherm geometry of the warm pool. In section 2, scale invariance will be examined with using WOCE/TOGA surface drfiter data from 1990 to 1991 . In section 3, diffusivity in the western tropical Pacific warm pool region will be estimated using the velocity variance and intergral time scale from the drifter path data. Section 4, states the discussion and summary.

\section{Scale invariance in drifter paths}

\subsection{Data set}

Since 1988, the WOCE/TOGA Global Surface Velocity Program (SVP) has been organized to deploy and interpret data from Lagrangian drifters, which consist of a surface buoy tethered to a drogue in the mixed layer. The drifters were tracked with the Service Argos system, with position rms error being less than $200 \mathrm{~m}$ in latitude and $300 \mathrm{~m}$ in longitude.

We obtained position data for SVP Lagrangian drifters deployed in the Pacific ocean west of date line from 1990 to 1991 . The data we analyzed were SVP surface drifter paths data for the periods from June 1990, to January 1991, from September 1991 to December 1991, and from September 1992 to December 1992.

We selected drifter paths for 14 drfiters, out of 239 drifters, which stayed for more than 60 days in the area of the tropical Pacific from 140E to $180 \mathrm{E}$, and from the equator to $5 \mathrm{~N}$ (Figure 1). Positions of the drifters were

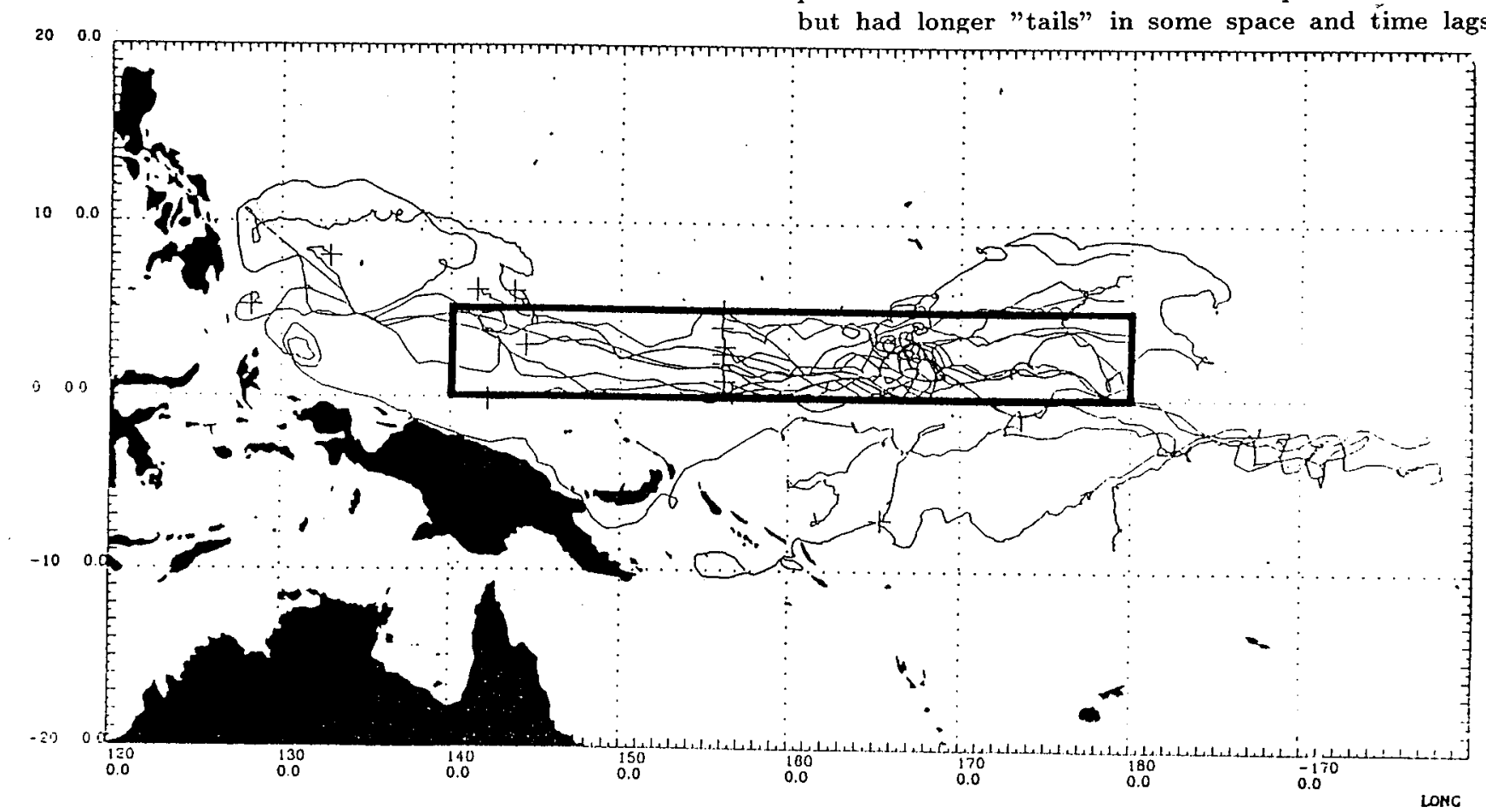

determined several times per day by the Service Argos system, and the sampling interval was 9.24 hours on the average. The drifter positions were interpolated to 6 hours by a cubic-spline interpolation scheme, and then the interpolated position records were used to estimate instantaneous velocities using forward differences of the interpolated positions. Mean velocities were defined by the total displacement, for the entire selected domain, devided by time period from the time history of the velocity series.

\subsection{Results of statistical analysis}

As may be seen from Figure 1, the present data represent measures of oceanic motions zonally scattered rather than concentrated in space. Decomposition of velocities into time average and fluctuating quantities yields mean zonal velocity of $0.3 \mathrm{~m} / \mathrm{sec}$ and mean meridional velocity of $0.01 \mathrm{~m} / \mathrm{sec}$. This is important from a statistical viewpoint because spatial inhomogenieties are then compelled to play a major role.

In Figure 2, the correlation functions obtained from 14 drifter paths are shown. The solid line is the mean estimator for correlation function from the 14 drifter paths and the upper and lower broken lines represent the standard deviation. It may be noted that the eddy fluctuations are asymmetric in the sense that the zonal de-correlation time is longer than the meridional de-correlation time. The zonal correlation function decreases up to 5 days time lag, and it has a "long tail" plateau between 5 days to 10 days. Similar results have been reported in the analysis of oceanic precipitaion patterns in which the spatial and the temporal auto-correlations follow near-exponential forms, but had longer "tails" in some space and time lags

Figure 1. Composit drifter paths for WOCE/TOGA Surface Velocity Program(SVP) during the period of 1990-1991. 
(Zawadzki, 1973; Nakamoto et al., 1990). Naturally, oceanic rainfall may play an important role in the upper ocean dynamics in the tropical ocean, and similarity in correlation functions between precipitation and Lagrangian float trajectories was not surprising.

On the other hand, the meridional correlation function decreases more rapidly than the zonal correlation function, and has a zero- crossing at 5 days, and asymptotes to zero at 15 days time lag. The zerocrossing was influenced by motions that have oscillations at about 10 days period, perhaps due to energy containing eddy fluctuation.

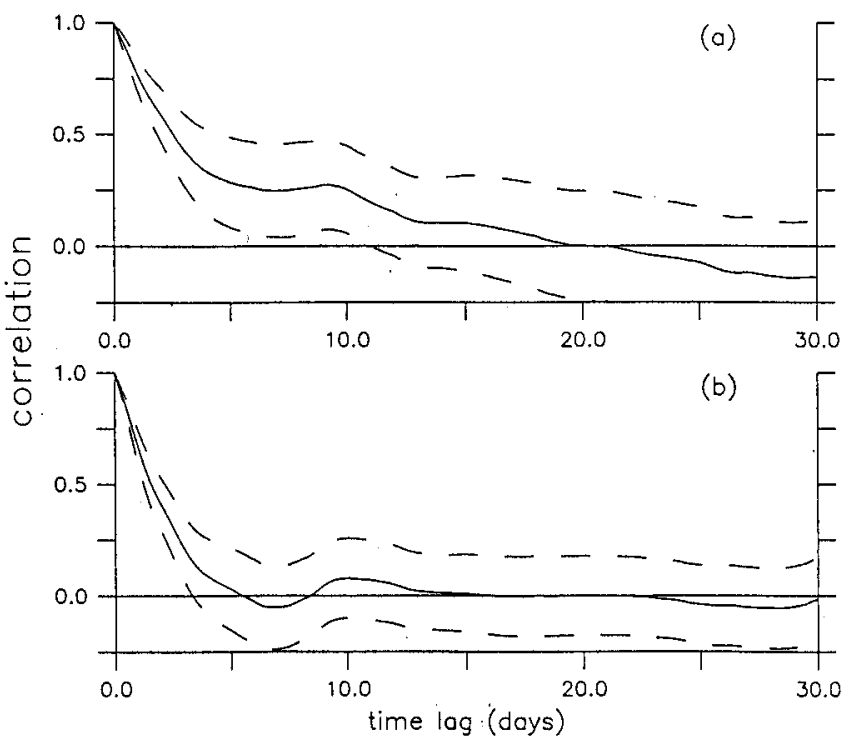

Figure 2. Average Lagrangian auto-correlation function from drifters for zonal velocity (a), and for meridional velocity $(b)$. The solid line is the mean estimator from 14 drifters and the upper and lower broken lines represent the standard deviation.

\subsection{Scale invariance in drifter paths}

Scaling notions are associated with lack of charcteristic scales over wide ranges, and the appearance of fractal dimensions and structures. More precisely, a system is said to be scaling (or scale invariant) over a range if the small and large scale structures are related by a scale changing operation that involves only the scale ratio. The result that the Brownian record looks "the same" under a change of resolution is called a scale invariance or symmetry of the Brownian record. In this study we search for the existence of such scale invariance in the upper ocean Lagrangian data and examine its relevance to a self-similar cascade of energy from large to small scales. The scaling exponent computation method used here is the same as that employed by Provenzale et al.[1988] and explained in the Appendix.

In Figure 3, the scale invariances for 14 drifters in the zonal path process $x(t)$ and in the meridional path process $y(t)$ are shown. It seems that scaling exists up to $10 \Delta t$, or 60 hours both in the zonal direction and in the meridional direction, however, the change in slope for a small range of $\Delta t$ is too small to identify the lin-
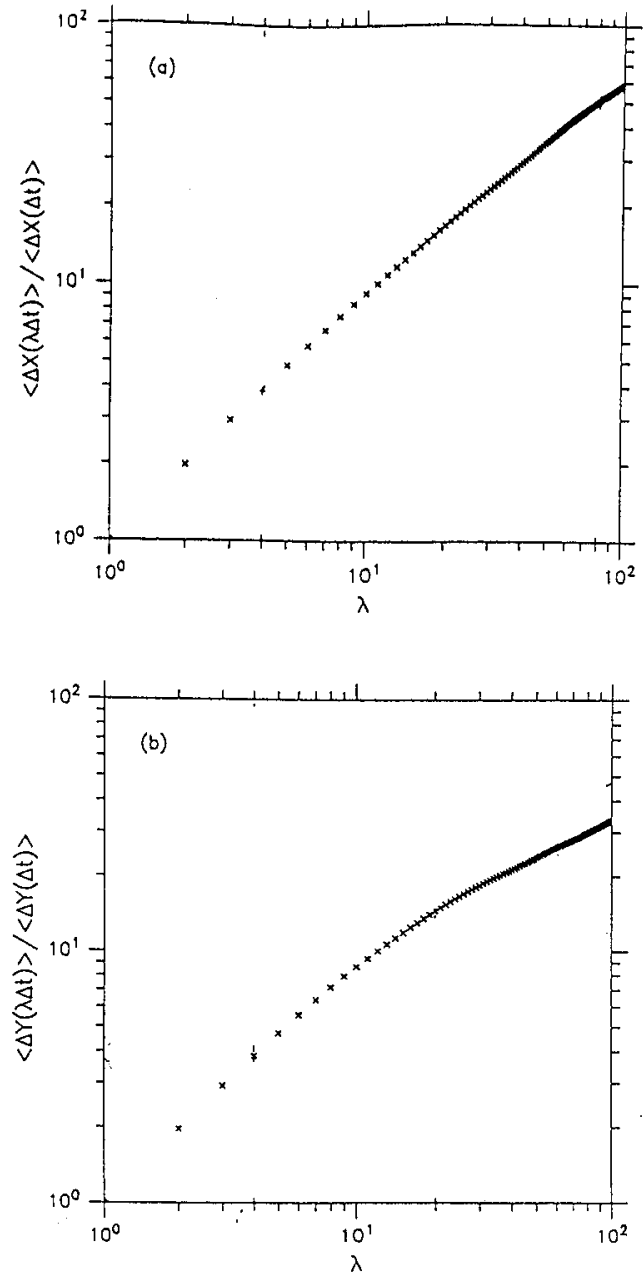

Figure 3. Plot(a) of $\langle x(\lambda \Delta t)-x(t)\rangle v s . \lambda$ and plot(b) of $\langle x(\lambda \Delta t)-x(t)\rangle$ vs. $\lambda$ in double logarithmic coordinates for the 14 drifter paths.

earity. The value of the scaling exponent $\mathrm{H}$, defined by the slope between two neighboring points separated by $\Delta t$, is plotted in Figure 4. It is noted that the scaling exponent functions in Figure 4 have similar shapes to those of the temporal correlation functions of Figure 2: the zonal scaling exponent has a "long tail" between 5 days to 10 days, and the zonal correlation function has a similar "long tail" between the same region in time lag, while the meridional scaling exponent asymptotes to 0.5 at 5 days, and the meridional correlation function asymptotes to zero at about 5 days. The meridional motion can be well approximated by Brownian processes with scaling exponent $H=0.5$. Since there was no ambient mean flow in the meridional direction, the drifter diffusion must be mostly due to eddy fluctuations meridionally.

On the other hand, there exist both mean and fluctuating flows zonally. By multiplying the zonal mean velocity of $U=0.3 \mathrm{~m} / \mathrm{sec}$ with the above mentioned scaling time range (from 5 days to 10 days), we obtain the corresponding zonal scaling range (from 130 $\mathrm{km}$ to $260 \mathrm{~km}$ ). The "long tail", representing deviation from exponential dacay of Markovian process cor- 
relation function, may be relevant to understanding fractional Brownian motion, having scaling range corresponding with that of the above "long tail".

These scale ranges agrees with the maximum length scale of fractal and scaling of drifter trajectories in the Kuroshio extension region [Osboren et al., 1989] and in the Northeast Altamtic [Sanderson and Booth, 1991]. It is interesting that the zonal mean velocity of 0.3 $\mathrm{m} / \mathrm{sec}$ is close to the Osborne et al.'s value $0.2 \mathrm{~m} / \mathrm{sec}$ that was considered to be a turbulent intensity and was in general consistent with usually accepted bounds on geostrophic or quasi-geostrophic turbulence [Osborne et al., 1989]. In the next section the above scaling exponent will be examined in light of spreading of warm water, and a single length scale from steady state diffusion system will be introduced to be components of fractal Brownian processes.
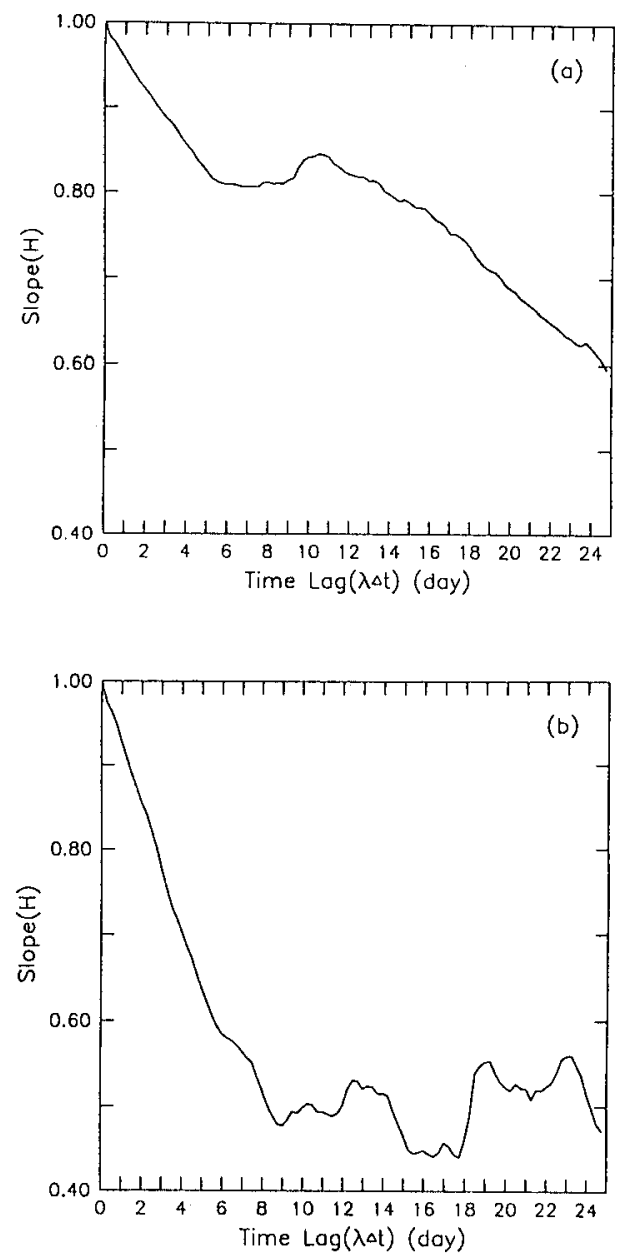

Figure 4. The slopes in zonal direction(a) and in meridional directionm (b) of the graphs in Figure 3 are plotted.

3. West tropical Pacific warm pool system

\subsection{Introduction}

Lagrangian dispersion analyses are usually based on the original papers of Taylor [1921] in which an asymp- totic law was found for the dispersion of particles from a fixed origin and for the relative dispersion of particles under the assumption of homogeneity and stationarity of the underlying turbulent field. Once the mean velocity was subtracted from the original velocity time series, the fluctuating velocity time series may be treated as irregular events, although our data set contains the time period of El Nino-like events. In this sense, we consider that the El Nino-like events in our data set may be manifestations of a nonlinear dynamical system in the west Pacific warm pool region as is the case of intermittency in three dimensional turbulence.

3.2 Anomalous diffusivity and the west Pacific warm pool

To show the diffusive character of the surface currents, the changes in meridional and zonal distance from their initial positions of the drifters are plotted in Figure 5 as a function of elapsed time since deployment. The number of independent trajectories has been increased from 14 to 110 by assuming that trajectories following a single drifter are uncorrelated after 5 days. This method of creating "pseudo-trajectories" was also adopted by Colin de Verdiere [1983], Poulain and Niiler [1987], and Paduan and Niiler [1993].

The single particle diffusivity is defined as the time rate of dispersion about the mean trajectory of a parcel. From velocity time series, we calculated the variance of velocity and the integral time scale by integrating time correlation function to obtain difusion coefficient of $41000 \mathrm{~m}^{2} / \mathrm{sec}$ zonally, and $8100 \mathrm{~m}^{2} / \mathrm{sec}$ meridionally.

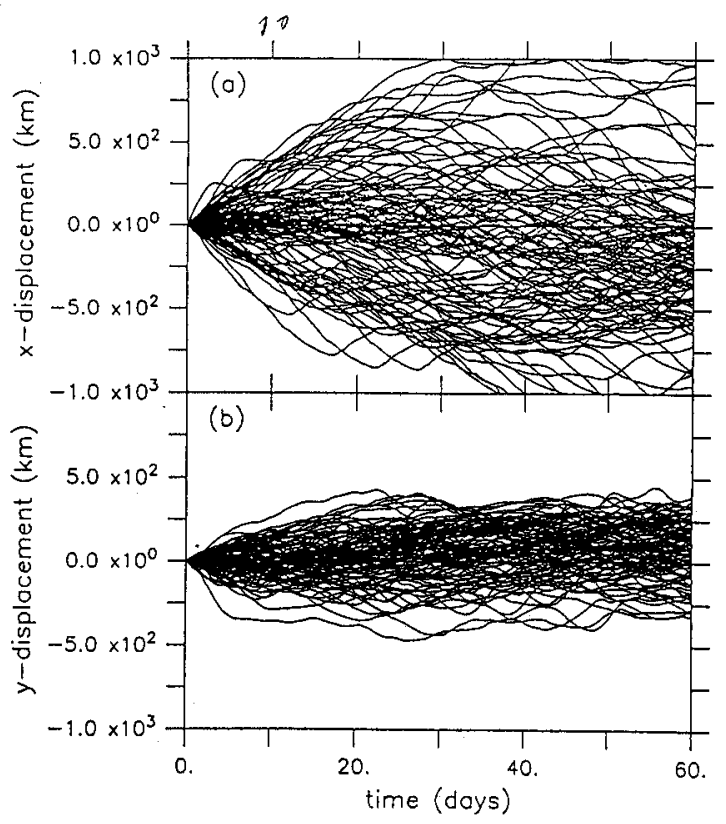

Figure 5. Dispersion away from initial location for 110 pseudotrajectories created from 14 original trajectories by assuming particle paths are uncorrelated after 5 days. 
The values we obtained are larger than values obtained in other parts of the world ocean. For instance, using Lagrangian surface drifter data, Paduan and Niiler [1993] estimated diffusivities to be $1600 \mathrm{~m}^{2} / \mathrm{sec}$ meridionally and $1100 \mathrm{~m}^{2} / \mathrm{sec}$ zonally in the north east Pacific. Using satellite-tracked drifter path data in the North Atlantic, Krauss and Boning [1987] showed that eddy diffusivity increases from $30 \mathrm{~N}$ to $50 \mathrm{~N}$ by a factor of about 4 . They obtained diffusivity values of 4500 $\mathrm{m}^{2} / \mathrm{sec}$ meridionally and $6600 \mathrm{~m}^{2} / \mathrm{sec}$ zonally. They also showed that the energy containing frequency band of the eddies shifts towards higher frequencies in the northern part of the Atlantic. In their case, eddies are of approximately equal size in the North Atlantic between $30 \mathrm{~N}$ and $50 \mathrm{~N}$, but energy increases in these eddies with increasing latitude. Consequently, a particle needs less time to surround a strong eddy, yielding shorter time scales and shorter diffusivites in the north.

If we assume eddy length scales, in the west $\mathrm{Pa}$ cific warm pool region and in the mid-latitudes, are characterized by the local Rossby deformation radii, we may interprete the anomalously large meridional diffusivity in our data set: the ratio of two deformation radii between $5 \mathrm{~N}$ and $50 \mathrm{~N}$ is 8 , which is 1.5 times of the ratio of two meridional diffusivities between $5 \mathrm{~N}$

and $50 \mathrm{~N}$. We feel that capturing irregular eddy motion having the local deformation length scale in the west tropical Pacific may be responsible for the meridional diffusivity.

The anomalous diffusivity in the zonal direction may be interpreted in relation with fractional Brownian motion. In order to demonstrate this, we compare position variance from Lagrangian drifter path data with that predicted by fractional Brownian processes.

The fractional Brownian process has zero average increments, and a variance of increments of the processes is given by:

$$
<[x(t)-x(0)]^{2}>=t^{2 H},
$$

where $x(t)$ represents the parcel location at time $t$ [Mandelbrot and Van Ness, 1968; Sanderson and Booth, 1991]. The above relation shows that fractional Brownian particle with $\mathrm{H}>1 / 2$ moves anomalously large distance from the origin over a finite range of physical scales, as compared with ordinary Brownian motion. Figure 6. shows the variance for artificially added drifter paths. For meridional paths, the variance follows ordinary Brownian type diffusion, which leads the square dependence for small time and linear dependence after decorrelation time. However, the variance for zonal paths showed different characteristics from that of the meridional ones. The variance has square dependence for small time. From 5 days to 10 days, when the correlation has long tail and scaling exponent of constant value $\mathrm{H}=0.7$, the variance has the power of

1.4 , as shown by the above equation. This means that the zonal diffusion for a time range between 5 days to 10 days may be governed by fractional Brownian type diffusion.

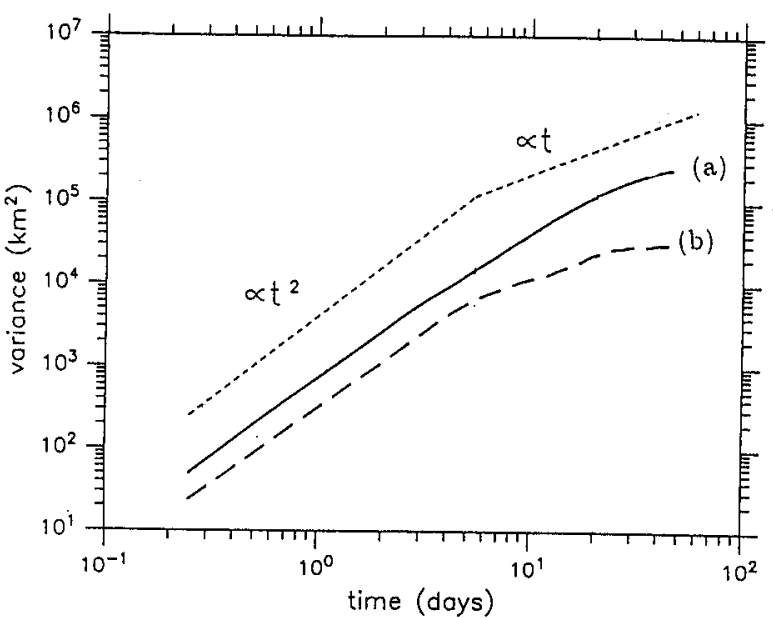

Figure 6.The position variance plotted logarithmically as a function of time for zonal direction (a), and for meridional direction(b). (a) breaks a Brownian process diffusion from 5 days to 20 days, while (a) reveals Brownian processes upto 20 days.

\subsection{Scale invariance in the west Pacific warm pool}

We seek scale invariance in the context of dynamical interpretation of the west Pacific warm water pool. Our motivation is to see if we can interprete the existence of a range in which the system can choose any length scale. In such a scale range, the system bahaves as if it excutes self-similar energy cascade turbulent processes rather than ordinary Brownian processes. We feel that self-similar energy cascade processes may possiblly explain the existence of scaling invaliance in the warm pool region: the irregular eddy motion in the warm pool region is fed and drained by large scale mean flow [Wyrtki, 1988] which seems irregular and unpredictable.

If the Lagrangian drifter tracking represents water particle motion in the warm pool, and if the water particle position is governed by increment stationary processes, then observers can not distinguish short time increment from long time increment of Lagrangian drifter paths. In the range of constant scaling exponent, one can never distinguish the characteristic time scale, or equivalent length scale in the range of $130 \mathrm{~km}$ and $260 \mathrm{~km}$.

This can be understood by re-writting classical diffusion equations into the staedy diffusion equation in

the moving coordinate system with mean flow speed, since the steady state equations represent time scale invariance: one can not trace time history of the diffusion in the fractal/scaling ranges.

Let the sea surface temperature of the tropical Pacific warm pool be described by the following zonal diffusion equations in the moving coordinate system as:

$$
\frac{\partial T}{\partial t}=v_{0} \frac{\partial T}{\partial x}+D_{x} \frac{\partial^{2} T}{\partial x^{2}}+D_{y} \frac{\partial^{2} T}{\partial y^{2}}+D_{z} \frac{\partial^{2} T}{\partial z^{2}},
$$

where $x$ is the zonal direction of the isotherm propaga- 
tion, $y$ is perpendicular to $x$, and $z$ is the vertical direction. The vertical diffusivity $D_{z}$ is small compared with horizontal diffusivities, $D_{x}$ and $D_{y}$. The first term in the left hand side represents advection by zonal mean curent velocity $v_{0}$. The steady state solution satisfies the above equation with the left side set to be zero. The above equation can be written as

$$
0=\frac{\partial}{\partial x}\left(\frac{\partial T}{\partial x}\right)+\frac{1}{l_{D}} \frac{\partial T}{\partial x}+\frac{D_{y}}{D_{x}} \frac{\partial^{2} T}{\partial y^{2}},
$$

where $l_{D}=D_{x} / v_{0}$ and the vertical diffusion term was neglected compared with horizontal diffusion terms. Using the diffusivities computed from the integral time scales for drifter paths time records, the ratio of the meridional diffusivity and the zonal diffusivity was computed to be $D_{y} / D_{x}=0.2$. Neglecting this term the above equation has a single length scale $l_{D}$. Using the zonal diffusivity of $41000 \mathrm{~m}^{2} / \mathrm{sec}$ from the drifter path analyses, and an advection velocity of $0.3 \mathrm{~m} / \mathrm{sec}$ from the

mean Lagrangian drifter speed in the zonal direction, we obtain $l_{D}$ to be $136 \mathrm{~km}$. This means that the ensemble of the suspended matter behaves as if it were governed by the fractal system with the upper bound being $136 \mathrm{~km}$. When zonal length scale becomes $l_{D}$, the ensemble of Lagrangian drifter paths may be regarded as a spreading substance which is governed by the above Brownian type diffusion equation. We can interpret the self-similarity in Lagrangian paths as an approximation of an accumulation of any number of consecutive occurences of the frcatal process with the upper bounded length scale of $136 \mathrm{~km}$.

For time scales upto 5 days to 10 days, or equivalently, for length scales upto $136 \mathrm{~km}$ to $270 \mathrm{~km}$, each realization of Lagrangian drifter may take any values and in this sense there exist no specific scale for each realization. Each paths looks ramdom realization to observers; however one part of paths is an exact copy of the other part with constant scaling exponent of $H=0.7$. These essemble can be statistically described by steady state diffusion equations with a single length scale approximately $130 \mathrm{~km}$.

\section{Disscussion and Summary}

We have shown that there exists a scale invariance in the west tropical Pacific drifters deployed in the WOCE/TOGA surface velocity program. The drifter paths in the zonal direction showed a range of scale invariance, with approximate scaling exponent of $\mathrm{H}=0.7$, upto 5 days to 10 days, while drifter paths in the meridional direction indicated ordinary Brownian motion after 5 days with approximate scaling exponent of $\mathbf{H}=\mathbf{0 . 5}$. Using the mean translation velocity of the ensemble of drifters $(0.3 \mathrm{~m} / \mathrm{sec}$ zonally and $0.01 \mathrm{~m} / \mathrm{sec}$ meridionally) the above scaling ranges corresponded with upto $130 \mathrm{~km}$ to $260 \mathrm{~km}$ zonally and several kilometers meridionally.

With using Taylor's classical turbulent regime, the particle paths variance and diffusivity are computed, and these values are compared with the equivalents predicted from fractional Brownian processes.

Using velocity variance and integral time scale obtained from WOCE/TOGA surface drifter tracking, meridional and zonal diffusivities were computed. These values were anomalously large compared with those obtained from drifter paths in mid-latitudes of the world ocean. However, the variance and diffusivity agreed with those predicted, by the fractional Brownian process zonally, and by Brownian processes meridionally. Since the diffusivities were computed by using velocity variance and integral time scale, the magnitudes of diffusivities may be strongly influenced by the estimation of the magnitude of the ambient mean flow. In this sense we may reserve concluding remarks on anomalous diffusivities in the west tropical Pacific warm pool. However it was noted that the two differnt approachs identified the common scale range satisfying self-similarity and a "long tail" in auto correlation functions in our data sets. We hypothesized the anomalously large diffusivities to be a manifestation of fractional Brownian motion in the range between 130 $\mathrm{km}$ and $260 \mathrm{~km}$.

The mechanism of exhibiting scale invariance in the zonal time history of drfiter positions was further proposed by introducing diffusion equations with a single length scale, which was the accepted upper bound of geophysical turbulence and can be considered to be the upper bound of fractional Brownian processess. We speculate that the surface Lagrangian flow feature in the warm water pool may be related with a self-similar energy cascade processes from large scale mean flow to small scale irregular motion in the zonal direction.

\section{Acknowledgments}

The authors wish to acknowledge WOCE/TOGA Surface Velocity Program office for providing us the drifter data that were used in this study. Mr. Shinichi Ishii, excetutive director of Japan Marine Science and Terchnology Center has encouraged our study of predictability of Oceanography. S. Nakamoto expresses his sincere thanks to Professor A. D. Kirwan of Old Dominion University for his suggestion of the nonlinear dynamical problem and its application to oceanic observational data sets.

\section{Appendix}

We note that the scale invariance is not the same as the classical law of similarity in which the size of the region is determined by a length $\mathrm{L}$, and the velocity is based on a mainstream velocity, $V$. In the classical regime, all similar flows, sharing the same Reynolds number, can be found by rescaling the solution of nondimensional Navier-Stokes equations. Flows at different speeds past different locations and with different viscosity depends on the single number $V L / \nu$.

Suppose that at time $t=0$, the water parcel is located at the origin of the real line, and let $x(t)$ be the coordinate of the parcel at the time $t$. The random process $x(t)$ is obviously not stationary. However the set of paths traversed by the water parcel during consecutive and equal time intervals form a stationary random sequence.

Consider the increments of the process given by

$$
<x(\lambda \Delta t+t)-x(t)>=\lambda^{H}<x(\Delta t+t)-x(t)>,
$$


where $\lambda$ is arbitrary and $\Delta t$ is the minimum time lag between observations. The bracket means taking the ensemble average of the process. The stationarity means that the above increment is a function of the time lag $\lambda$ only. In the actual data analysis, the above ensemble average was replaced with the time average by generating different realizations shifted by the de-correlation time lag.

\section{References}

Colin de Verdiere, A., Lagrangian eddy statistics from surface drifters in the eastern North Atlantic J. Mar. Res., Vol. 41, 375-398, 1983

Feder J., Fractals, Plenum Press, New York, 233 Spring Street, New Yory, N.Y. 10013

Krauss, W., and C. W. Boning., Lagrangian properties of eddy fields in the northern North Atlantic as deduced from satellite-tracked buoys J. Mar. Res., Vol. 45, 259-291, 1987

Mandelbrot, B., and J. W. Van Ness, Fractal Brownian Motions, Fractal Noises and Applications SIAM Rev., Vol. 10, No.4, 422-437, 1968

McNally, G. J., Satellite-tracked buoy observations of the near surface flow in the eastern midlatitude north Pacific. $J$. Geophys. Res., Vol. 86, 8022-8030, 1981
Nakamoto S., J. B. Valdes, G.R.North, FrequencyWavenumber Spectrum for GATE Phase I Rainfields. J. Appl. Meteor., Vol. 29, 842-850, 1990

Niiler, P.P., R. E. Davis, and H. J. White., Waterfollowing characteristics of mixed layer drifter. Deep Sea Res., Vol. 34, 5094-5102, 1987

Osborne A. R., A.D.Kirwan, A. Provenzale and L. Bergamasco. Fractal drifter trajectories in the Kuroshio extension. Tellus , Vol 41A 416-435, 1989

Provenzale A., A.R.Osborne, A.D.Kirwan, and L. Bergamasco. The Study of Fluid Parcel Trajectories in Large-Scale

Ocean Flows. Nonlinear Topics in Ocean Physics, International Shcool of Physics < ENRICOFERMI > Course CIX, Italian Physical Society, North-Holland Elsevier Science Publishers B.V., P.O.Box 2111000 A E Amsterdam, The Netherland. 367-402, 1991

Sanderson B. G., and D. A. Booth The fractal dimension of drifter trajectories and estimates of horizontal eddydiffusivity Tellus, Vol 43A 334-349, 1991

Taylor, G.I., Diffusion by continuous mevements. Proc. Lond. Math., Vol. 7, 50-61, 1977.

Zawadzki, I., Statistical properties of precipitation patterns. J. Appl. Meteor., Vol. 12, 469-472, 1973 